\title{
A Comparative Study of Time Advancement Methods for Solving Navier-Stokes Equations
}

\author{
T. K. Sengupta ${ }^{1}$ and A. Dipankar ${ }^{1}$
}

Received May 28, 2003; accepted (in revised form) November 27, 2003

A qualitative and quantitative study is made for choosing time advancement strategies for solving time dependent equations accurately. A single step, low order Euler time integration method is compared with Adams-Bashforth, a second order accurate time integration strategy for the solution of one dimensional wave equation. With the help of the exact solution, it is shown that the presence of the computational mode in Adams-Bashforth scheme leads to erroneous results, if the solution contains high frequency components. This is tested for the solution of incompressible Navier-Stokes equation for uniform flow past a rapidly rotating circular cylinder. This flow suffers intermittent temporal instabilities implying presence of high frequencies. Such instabilities have been noted earlier in experiments and high accuracy computations for similar flow parameters. This test problem shows that second order AdamsBashforth time integration is not suitable for DNS.

KEY WORDS: DNS; time integration methods; dispersion relation preservation; Navier-Stokes equation; flow instabilities.

\section{INTRODUCTION}

Availability of very fast computing machines with large memory allows one to solve the Navier-Stokes equation for high Reynolds number flows, resolving all spatial and temporal scales by Direct Numerical Simulation (DNS). For DNS lots of attention has been given in devising high accuracy spatial discretization schemes. These schemes are essentially based on higher order explicit or implicit schemes for the discretization of non-linear convection terms. The explicit higher order upwind schemes have been variously used to study unsteady and turbulent flows in [1-3]. The implicit

${ }^{1}$ Department of Aerospace Engineering, I.I.T. Kanpur, U.P. 208016, India. E-mail: tksen@ iitk.ac.in 
methods, also known as compact schemes, are based on Pade' approximation. These are known to possess higher spectral accuracy than the explicit higher order upwind schemes. Some of the important and recent work in this area are to be found in [5-7]. However, in the present research the attention is solely focused on explicit methods. In particular, we will consider the third order upwind scheme [1-3] that discretizes the convection term as

$$
\begin{aligned}
\left.f \frac{\partial u}{\partial x}\right|_{i}= & \frac{f_{i}}{12 \Delta x}\left[-u_{i+2}+8 u_{i+1}-8_{i-1}+u_{i-2}\right] \\
& -\beta \frac{\left|f_{i}\right|}{12 \Delta x}\left[u_{i+2}-4 u_{i+1}+6 u_{i}-4 u_{i-1}+u_{i-2}\right]
\end{aligned}
$$

In this expression on the right hand side, the second term is an extra additive term that numerically stabilizes computation. Here a fourth derivative term is blended with the discrete first derivative term (the first term on the right hand side), with the help of a blending parameter, $\beta$. The idea that this method stabilizes the computations, stems from the fact that the second set of terms in Eq. (1) provides a negative feed back. It is noted that this used even derivative terms are added in such a way that the corresponding difference expression for the evolution of the node value $u_{i}$ has a negative sign for the coefficient on right hand side. Thus, when a second derivative is used it is always added, while the fourth derivative term is always subtracted. A direct connection of higher order upwind methods have been shown [8] with Large Eddy simulations (LES). The implicit dissipation term was shown in [8] to be equivalent to the sub-grid scales stress models used in LES.

While sufficient attention has been spent on the spatial discretization, there has been lesser focus on the time discretization schemes. For example, in [1-3] a first order accurate Euler time integration scheme has been used. The marker and cell (MAC) method of [4] is an explicit method specifically designed for Euler time integration scheme. In the original MAC method, second order central differencing is used for spatial discretization. The SMAC method [1] used third order upwind scheme for spatial discretization and Euler time integration for integrating Navier-Stokes equation using primitive variables. It is expected that a choice of higher order accurate time integration will facilitate taking larger time steps and achieving higher accuracy of the solution. Some of the classical higher order time integration schemes continues to enjoy popularity in diverse application as in reactive flow computations [12] and geophysical fluid dynamics [13]. Adoption of Leap-frog (second order accurate) time integration scheme for diffusion equation leads to instability [9]. At the same time this scheme for 
the wave equation displays neutral stability. This three time level marching scheme has two amplification factors $G_{1}^{*}$ and $G_{2}^{*}$, such that $\left|G_{1,2}^{*}\right|=1$ for all length scales if the CFL number $\left(N_{c}\right)$ is chosen such that, $N_{c} \sin (k \Delta x)<1$. The weather prediction codes that usually solve inviscid equations make use of this feature of Leap-frog method for accurate simulation. However, the simulation results tend to decorrelate with observation at large times due to aliasing errors, phase error and the effects of non-linearity of the governing equations. In [10] a complete analysis has been performed for this three level scheme to express general solution in terms of $G_{1}^{*}$ and $G_{2}^{*}$. The first part of the solution (called the physical mode) approaches the exact solution, while the second part of the solution (called the computational mode) approaches zero as, as $\Delta x$ and $\Delta t$ are allowed to approach zero. This is a spurious mode and a source of error of many simulations. It has been noted [10] that this mode alternates in sign and travels in the opposite direction to the physical mode. In the present work the attention is focused on time advancement schemes and not on spatial discretization scheme. Here either a second order or a fourth order accurate spatial discretization scheme is considered and the second order accurate AdamsBashforth time integration scheme is compared with first order accurate Euler time integration scheme. This is done to highlight the role of computational mode of high order explicit time integration schemes, as compared to the Euler time integration scheme that does not have this artificial mode.

Lilly [11], while examining time advancement scheme for integration of simplified form of barotropic vorticity equation, noted that second order Adams-Bashforth scheme performs best considering efficiency and accuracy. For a typical ordinary differential equation

$$
\frac{d A}{d t}=F(A, t)
$$

the Adams-Bashforth scheme for time-advancing the above equation from $t_{n}$ to $t_{n+1}$ is given by

$$
A\left(t_{n+1}\right)=A\left(t_{n}\right)+\frac{\Delta t}{2}\left[3 F\left(t_{n}\right)-F\left(t_{n-1}\right)\right]
$$

It has been noted in [10] following the results of [11], that the computational mode given by $G_{2}^{*}$ is a heavily damped mode. It is to be highlighted that by itself this is a desirable property. However, without elaboration it was also stated that the Adams-Bashforth scheme is suitable unless the period of integration is lengthy [10]. Because of this lack of clarity of this statement many researchers continue to use Adams-Bashforth scheme for DNS and acoustics problem that necessarily involves computing for long 
time. The main focus of the present work is to establish the unsuitability of Adams-Bashforth time integration scheme in producing accurate results for long time integration of evolutionary equations.

The use of Adams-Bashforth time integration scheme is very widespread, specially in recent times, because of its dissipative property, as compared to other lower order explicit time marching schemes like Euler time marching scheme. For example DNS of channel flow is reported in [14] that used Adams-Bashforth time integration along with second order central differencing for spatial discretization. Turbulent flow and heat transfer problem by DNS is reported in $[15,16]$, that used Adams-Bashforth scheme for time marching. In [16] turbulent flow over wavy boundary is solved using DNS and LES that used Adams-Bashforth scheme for time integration. Even for spectral methods [17] Adams-Bashforth scheme has been used to obtain high accuracy for temporal discretization. A few other representative references that used the same temporal discretization are as in [18-24]. In [25], free surface flow in open channel has been studied by DNS using Adams-Bashforth scheme. Such wide usage of Adams-Bashforth scheme for DNS and computational aero-acoustics prevails as there are no recorded proper analysis of Adams-Bashforth time integration scheme in the literature. With this in mind we thoroughly analyze the second order accurate Adams-Bashforth time integration scheme to find out limitations on wave numbers and circular frequencies for this scheme and suggest alternatives. Similar problems will be present for other explicit higher order time integration schemes involving computational mode. For example, the Runge-Kutta schemes are higher order schemes but they do not have computational mode and hence they will be perfectly usable for time dependent problems. The present study is undertaken to clearly assess the Adams-Bashforth time integration scheme vis-a-vis the Euler time integration scheme for the purpose of displaying the bad effects of computational mode whenever it is present. In the next section we investigate these two time integration schemes for different explicit spatial schemes for the onedimensional wave equation. We will use the spectral analysis tool that is developed in [26] for analyzing any general discretization scheme. In Sec. 2, a specific example of a flow past a rotating cylinder is considered, to pin-point the effectiveness of these time integration strategies.

\section{ANALYSIS OF ONE-DIMENSIONAL WAVE EQUATION}

Consider the one dimensional wave equation

$$
\frac{\partial u}{\partial t}+c \frac{\partial u}{\partial x}=0
$$


for which the initial solution $u_{0}(x)$ travels to the right at the phase speed $c$. For the numerical solution of Eq. (4), we identify the solution as

$$
U\left(x_{m}, t_{n}\right)=U_{m}^{n}=\int B\left(k, t_{n}\right) e^{i k x_{m}} d k
$$

\subsection{Euler Time Integration Scheme}

If we define CFL number as $N_{c}=c \frac{\Delta t}{\Delta x}=\frac{\omega \Delta t}{k \Delta x}$, then for the solution of Eq. (4) by Euler time integration and second order spatial discretization, the amplification factor, $G_{2}(k)=\frac{B\left(k, t_{n+1}\right)}{B\left(k, t_{n}\right)}$ can be obtained as $G_{2}(k)=(1+$ $\left.N_{c}^{2} \sin ^{2} k \Delta x\right)^{1 / 2} e^{-i \beta_{2}}$, where $\tan \beta_{2}=N_{c} \sin k \Delta x$. Thus if the initial solution is indicated by

$$
U_{m}^{0}=\int A_{0}(k) e^{i k x_{m}} d k
$$

then the general solution at any arbitrary time can be written as

$$
\begin{aligned}
U_{m}^{n} & =\int A_{0}(k)\left[G_{2}(k)\right]^{n} e^{i k x_{m}} d k \\
& =\int A_{0}(k)\left[1+N_{c}^{2} \sin ^{2} k \Delta x\right]^{\frac{n}{2}} e^{i\left(k x_{m}-n \beta_{2}\right)} d k
\end{aligned}
$$

$\beta_{2}$ gives a measure of the phase speed for the numerical scheme. Thus the numerical phase speed is given by $c_{N}=\frac{\beta_{2}}{k \Delta t}$ and the ratio $\frac{c_{N}}{c}=\frac{\beta_{2}}{\omega \Delta t}$ is the appropriate non-dimensional ratio that will be used. In Fig. 1(a) the contours for $G_{2}$ in the $(k \Delta x-\omega \Delta t)$-plane are plotted. It is evident that large range of $k \Delta x$ have the desired property of $G_{2}=1$, when $\omega \Delta t$ vanishes. Otherwise, the unstable nature of the scheme is self-evident. In Fig. 2(a) the region where $\frac{c_{N}}{c}$ is between 0.95 and 1 is shown as the shaded region. Ideally, one would like this ratio to be 1 and for the present scheme it is achievable only for the point at the origin. Here we show a region where a $5 \%$ error is tolerated. If one replaces the second order spatial discretization scheme by the fourth order scheme, i.e., using the following for the spatial derivative,

$$
\left(\frac{\partial u}{\partial x}\right)_{m}=\frac{1}{12 \Delta x}\left[-u_{m+2}+8 u_{m+1}-8_{m-1}+u_{m-2}\right]
$$


then one can obtain the amplification factor as $G_{4}(k)=1-i \frac{N_{c}}{3}(4-\cos k \Delta x)$ $\times \sin k \Delta x$. The general solution at any arbitrary time is given by

$$
U_{m}^{n}=\int A_{0}(k)\left[1+\frac{N_{c}^{2}}{9}(4-\cos k \Delta x)^{2} \sin ^{2} k \Delta x\right]^{\frac{n}{2}} e^{i\left(k x_{m}-n \beta_{4}\right)} d k
$$

where $\tan \beta_{4}=\frac{N_{c}}{3}(4-\cos (k \Delta x)) \sin (k \Delta x)$. In Fig. 1(b), $\left|G_{4}\right|$-contours are plotted in the $(k \Delta x-\omega \Delta t)$-plane. The contours are qualitatively the same as shown for $\mathrm{CD}_{2}$-case, i.e., the scheme is unstable but the level of instability is marginally lesser. In Fig. 2(b) the corresponding phase portrait of $\frac{c_{N}}{c}$ is shown where it takes the value between 0.95 and 1 . This shaded region is

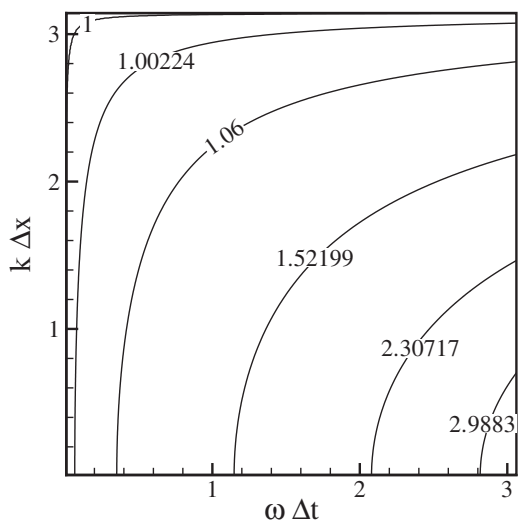

(a)

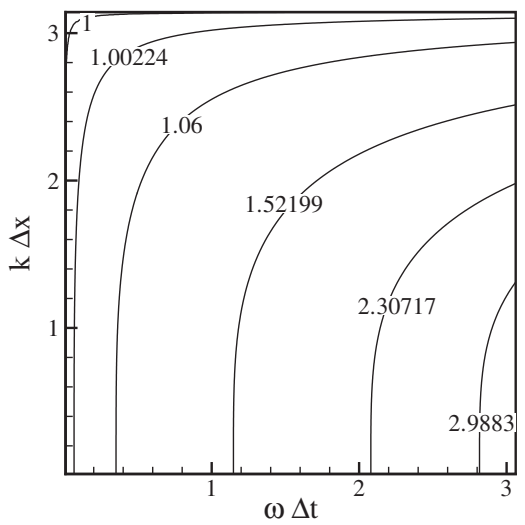

(b)



(c)

Fig. 1. The amplification factor in the full $(k \Delta x-\omega \Delta t)$-plane for Euler time integration scheme and spatial discretization by (a) $C D_{2}$, (b) $C D_{4}$, and (c) $U D_{3}$ schemes. 


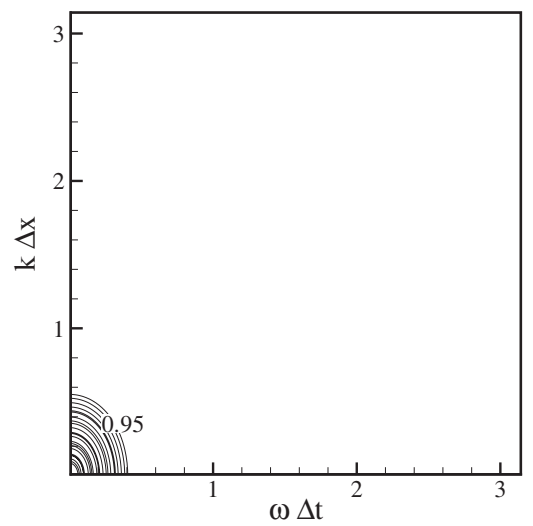

(a)

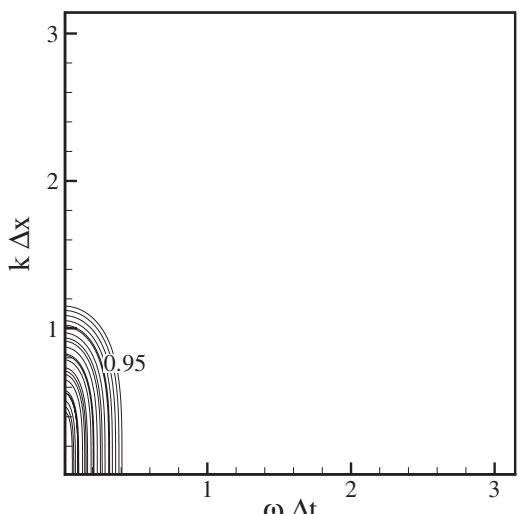

(b)

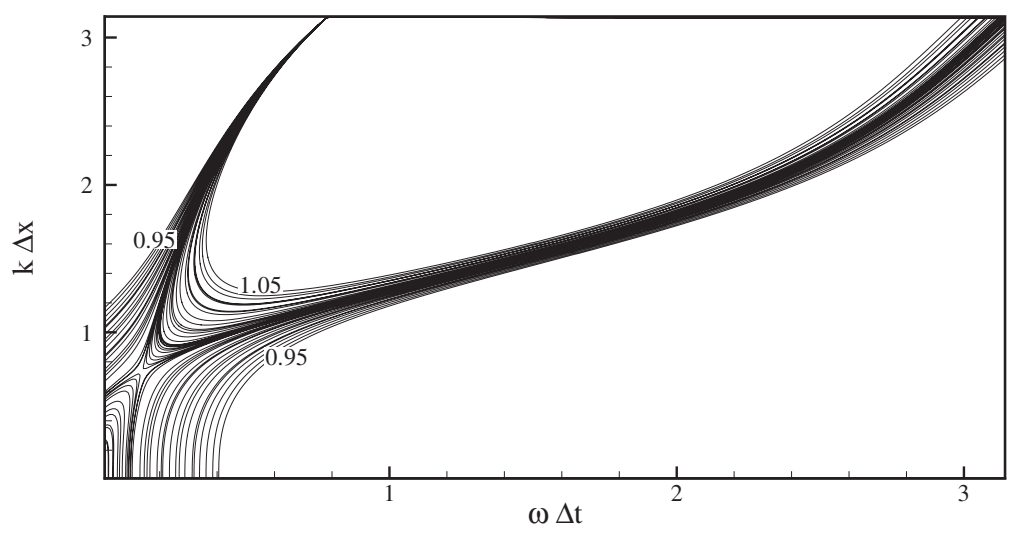

(c)

Fig. 2. The normalized numerical phase speed $\left(c_{N} / c\right)$ for solving Eq. (4) using Euler time integration scheme; (a) the shaded region where $0.95<\frac{c_{N}}{c}<1$ for $C D_{2}$ scheme; (b) the shaded region where $0.95<\frac{c_{N}}{c}<1$ for $C D_{4}$ scheme; and (c) where $\left|\frac{c_{N}}{c}-1\right|<0.05$ for $U D_{3}$ scheme for spatial discretization.

bigger-but it is qualitatively also same as that is obtained for $C D_{2}$ scheme. These known results are plotted to indicate the amplification and phase portraits of the schemes for all possible wave numbers and circular frequencies.

Next, if we replace the fourth order central difference scheme by third order upwind scheme [1-3], then the difference equation for Eq. (4) is

$$
\frac{U_{m}^{n+1}-U_{m}^{n}}{\Delta t}=-\frac{c}{6 \Delta x}\left[U_{m+2}^{n}-2 U_{m+1}^{n}+9 U_{m}^{n}-10 U_{m-1}^{n}+2 U_{m-2}^{n}\right]
$$


The corresponding amplification factor is given by

$$
G_{3}(k)=1-i \frac{N_{c}}{6}\left(L_{2}+i L_{1}\right)
$$

where $L_{2}=(4-\cos (k \Delta x)) \sin (k \Delta x)$ and $L_{1}=-24 \sin ^{2}\left(\frac{k \Delta x}{2}\right)$. The amplification rate can be rewritten as

$$
G_{3}(k)=\left(\left(1+\frac{N_{c}}{6} L_{1}\right)^{2}+\frac{N_{c}^{2} L_{2}^{2}}{36}\right)^{\frac{1}{2}} e^{-i \beta_{3}}
$$

where $\tan \beta_{3}=\left(\frac{N_{c} L_{2}}{6+N_{c} L_{1}}\right)$. Hence the general solution for the third order spatial upwind and Euler time discretization scheme is given by,

$$
U_{m}^{n}=\int A_{0}(k)\left[\left(1+\frac{N_{c}}{6} L_{1}\right)^{2}+\frac{N_{c}^{2} L_{2}^{2}}{36}\right]^{\frac{n}{2}} e^{i\left(k x_{m}-n \beta_{3}\right)} d k
$$

In Fig. $1(\mathrm{c}),\left|G_{3}\right|$-contours are plotted in the $(k \Delta x-\omega \Delta t)$-plane. This scheme is conditionally stable for the combinations of $k \Delta x$ and $\omega \Delta t$ in the upper left part of the $(k \Delta x-\omega \Delta t)$-plane. This figure explains why one requires a very small time step for stable computations. In Fig. 2(c) the shaded region depicts the values of $k \Delta x$ and $\omega \Delta t$ for which $\left|\frac{c_{N}}{c}-1\right| \leqslant 0.05$.

Apart from the amplification and phase portraits, one is interested in finding out the numerical group velocity of schemes. This is due to the fact that the energy of a convective system travels at the group velocity. This aspect of chosen numerical scheme in preserving dispersion relation is discussed in [26]. For the one-dimensional wave equation the physical group velocity is given by $c$. The numerical group velocity $V_{g N}$ can be found out from the numerical dispersion relation

$$
\omega_{e q}=c_{N} k
$$

by evaluating it from

$$
\frac{V_{g N}}{c}=\frac{c_{N}}{c}+\frac{k^{2}}{\omega} \frac{d c_{N}}{d k}
$$

The right hand side of the above expression can be calculated for any spatial and temporal discretization schemes for the one-dimensional wave equation. The results are displayed in Figs. 3(a)-(c) where $\frac{V_{g N}}{c}$ contours are plotted in $(k \Delta x-\omega \Delta t)$-plane for the above schemes. The shaded region in Fig. 3(a) is for $C D_{2}$-spatial discretization and one notices a small region near the origin where numerical dispersion relation is consistent with 
physical dispersion relation. This region increases for larger range of $k \Delta x$ for the $C D_{4}$-scheme. For the third order upwind scheme the corresponding region is shown in Fig. 3(c). Because of the complex numerical dispersion relation here we have an extended region as compared to the central schemes. In Figs. 2 and 3 , the $c_{N}$ and $V_{g N}$ plots indicate similar shaded region, but due to the dispersion relation the DRP region predicted by group velocity is smaller than that shown by the region where physical and numerical phase speeds are identical.

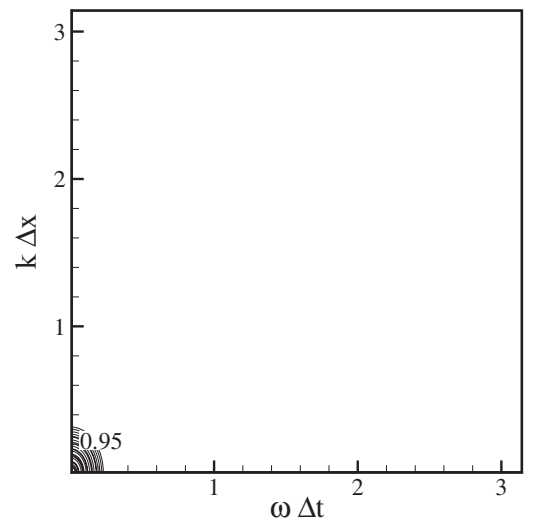

(a)

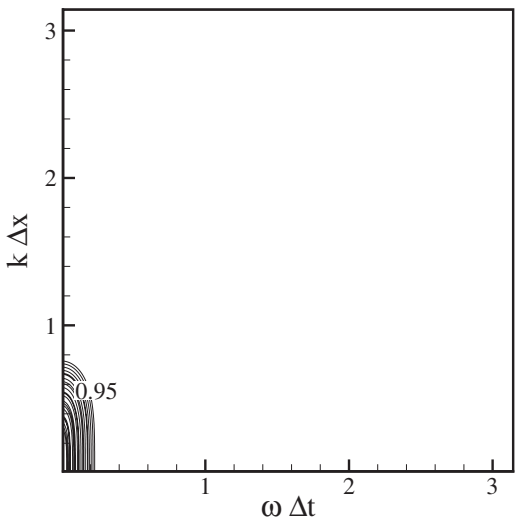

(b)

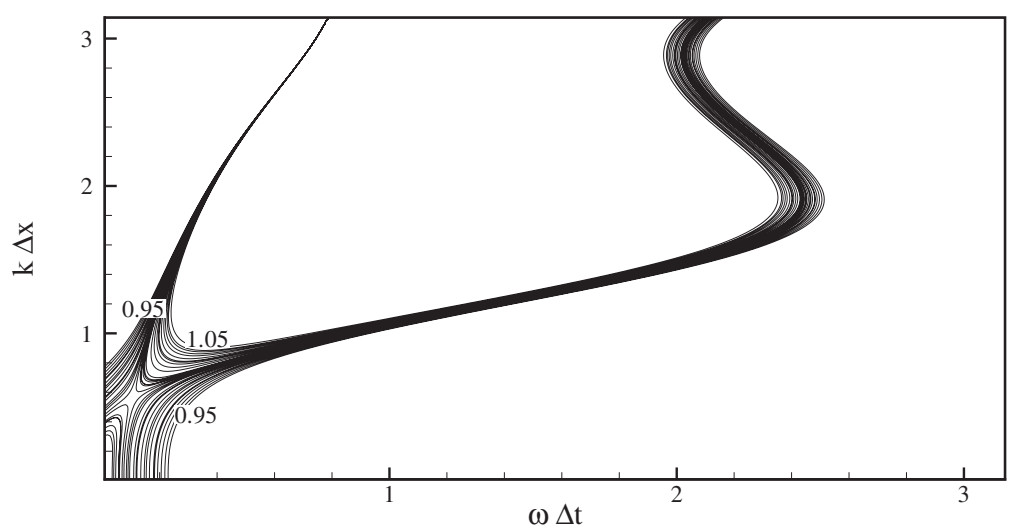

(c)

Fig. 3. The normalized numerical group velocity $\left(V_{g N} / c\right)$ for solving Eq. (4) using Euler time integration scheme; (a) the shaded region where $0.95<\frac{V_{g N}}{c}<1$ for $C D_{2}$ scheme; (b) the shaded region where $0.95<\frac{V_{g N}}{c}<1$ for $C D_{4}$ scheme; and (c) where $\left|\frac{V_{g N}}{c}-1\right|<0.05$ for $U D_{3}$ scheme for spatial discretization. 


\subsection{Adams-Bashforth Time Integration Scheme}

If we use the second order central differencing scheme for the spatial discretization along with second order Adams-Bashforth scheme (as given by Eq. (3)) the discrete equation for Eq. (4) is given by,

$$
\frac{U_{m}^{n+1}-U_{m}^{n}}{\Delta t}=-\frac{c}{2}\left[3 \frac{U_{m+1}^{n}-U_{m-1}^{n}}{2 \Delta x}-\frac{U_{m+1}^{n-1}-U_{m-1}^{n-1}}{2 \Delta x}\right]
$$

The amplification factors $G_{2}^{\mathrm{AB}}$ are the roots of the following quadratic equation,

$$
(G-1)+2 i \frac{N_{c}}{4} \sin (k \Delta x)\left(3-\frac{1}{G}\right)=0
$$

If the roots are indicated by $\lambda_{1}$ and $\lambda_{2}$ then

$$
\begin{aligned}
& \lambda_{1}=F_{2} e^{i \eta_{2}} \\
& \lambda_{2}=H_{2} e^{i \Gamma_{2}}
\end{aligned}
$$

where,

$$
\begin{aligned}
F_{2}(k) & =\left[C_{2}^{2}+D_{2}^{2}+2 C_{2} D_{2} \cos \left(\frac{\xi_{2}}{2}-\frac{\bar{\beta}_{2}}{2}\right)\right]^{\frac{1}{2}} \\
H_{2}(k)= & {\left[C_{2}^{2}+D_{2}^{2}-2 C_{2} D_{2} \cos \left(\frac{\xi_{2}}{2}-\frac{\bar{\beta}_{2}}{2}\right)\right]^{\frac{1}{2}} } \\
C_{2}= & \frac{1}{2}\left[1+\frac{9}{4}\left(N_{c} \sin (k \Delta x)\right)^{2}\right]^{\frac{1}{2}} \\
D_{2}= & \frac{1}{2}\left[1+\frac{81}{16}\left(N_{c} \sin (k \Delta x)\right)^{4}-\frac{7}{2}\left(N_{c} \sin (k \Delta x)\right)^{2}\right]^{\frac{1}{4}} \\
\tan \left(\bar{\beta}_{2}\right)= & -\frac{N_{c} \sin (k \Delta x)}{1-\frac{9}{4}\left(N_{c} \sin (k \Delta x)\right)^{2}} \\
\tan \left(\frac{\xi_{2}}{2}\right)= & -\frac{3}{2} N_{c} \sin (k \Delta x) \\
\tan \left(\eta_{2}\right)= & \frac{C_{2} \sin \left(\frac{\xi_{2}}{2}\right)+D_{2} \sin \left(\frac{\bar{\beta}_{2}}{2}\right)}{C_{2} \cos \left(\frac{\xi_{2}}{2}\right)+D_{2} \cos \left(\frac{\bar{\beta}_{2}}{2}\right)} \\
\tan \left(\Gamma_{2}\right)= & \frac{C_{2} \sin \left(\frac{\xi_{2}}{2}\right)-D_{2} \sin \left(\frac{\bar{\beta}_{2}}{2}\right)}{C_{2} \cos \left(\frac{\xi_{2}}{2}\right)-D_{2} \cos \left(\frac{\bar{\beta}_{2}}{2}\right)}
\end{aligned}
$$


The general solution at any arbitrary time is given by,

$$
U_{m}^{n}=\int M_{2}(k)\left(F_{2}\right)^{n} e^{i\left(k x_{m}-n \eta_{2}\right)} d k+\int N_{2}(k)\left(H_{2}\right)^{n} e^{i\left(k x_{m}-n \Gamma_{2}\right)} d k
$$

In Eq. (15) the first part is called the physical mode and the second part is the computational mode. Ideally, one expects the computational part to be negligibly small at all times. The multiplicative constants $M_{2}$ and $N_{2}$ can be evaluated from the conditions at $t=0$ (given by Eq. (6)) and at $t=\Delta t$ (to be obtained from Eq. (7) for $n=1$ ). Substitution and simplification yields,

$$
\begin{aligned}
& M_{2}(k)=A_{0} \frac{1-i N_{c} \sin (k \Delta x)-H_{2} e^{i \Gamma_{2}}}{F_{2} e^{i \eta_{2}}-H_{2} e^{i \Gamma_{2}}} \\
& N_{2}(k)=A_{0} \frac{-1+i N_{c} \sin (k \Delta x)+F_{2} e^{i \eta_{2}}}{F_{2} e^{i \eta_{2}}-H_{2} e^{i \Gamma_{2}}}
\end{aligned}
$$

For the Adams-Bashforth time integration scheme, there is no other way the time integration can be started without going through one step of Euler time integration. Thus the value of $M_{2}$ and $N_{2}$ obtained are unique in that sense. $M_{2}$ and $N_{2}$ indicate the fraction of initial condition shared between the physical and computational modes. In Figs. 4(a) and 4(b) contours of the time-dependent parts of the physical $\left(\mathrm{F}_{2}\right)$ and computational mode $\left(\mathrm{H}_{2}\right)$ are plotted in $(k \Delta x-\omega \Delta t)$-plane. It is evident that unlike the Euler time integration scheme, there is a larger range of $\omega \Delta t$ available for which the scheme is near-neutral for all wave numbers for the physical mode. Also, for the same range of $\omega \Delta t$ the computational mode is negligible. The corresponding spectral weights of the initial condition, $M_{2}$ and $N_{2}$, contours are plotted in Figs. 4(c) and 4(d) respectively. These demonstrate that the physical and computational modes taken together will produce stable results for very small $\omega \Delta t$ values. These figures also explain why Adams-Bashforth scheme is not workable for high accuracy computations. The computational mode by itself has the extremely nice property of being a highly damped mode. Thus its presence is not going to be felt within a few time step of integration. The problem originates from this source and the way the initial condition is partitioned. The initial condition that is used is divided between the physical and computational mode in the proportion dictated by $M_{2}$ and $N_{2}$. However, the quantity that is carried by $N_{2}$ is lost within a few time steps due to the highly damped nature of the computational mode. This loss of initial condition-information will drastically alter the numerically obtained solution by the Adams-Bashforth time integration scheme, as compared to the Euler time integration scheme. The latter does not have any computational mode and hence does not suffer from associated problem. 


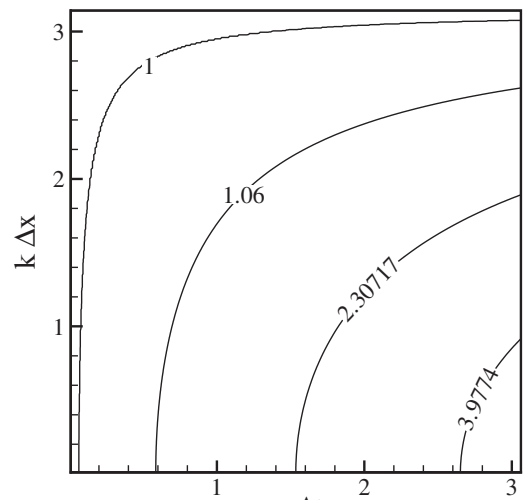

$\omega \Delta \mathrm{t}$

(a)

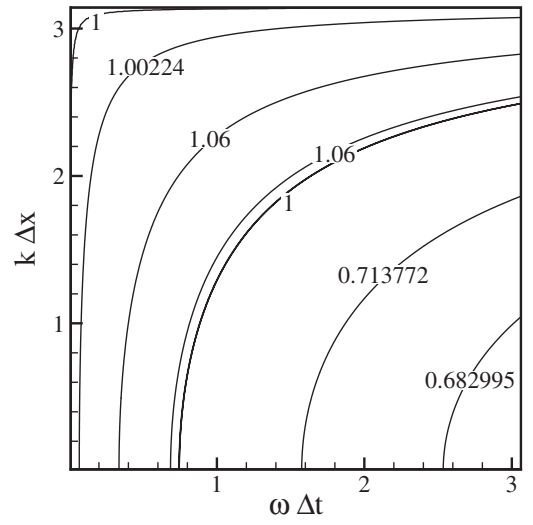

(c)

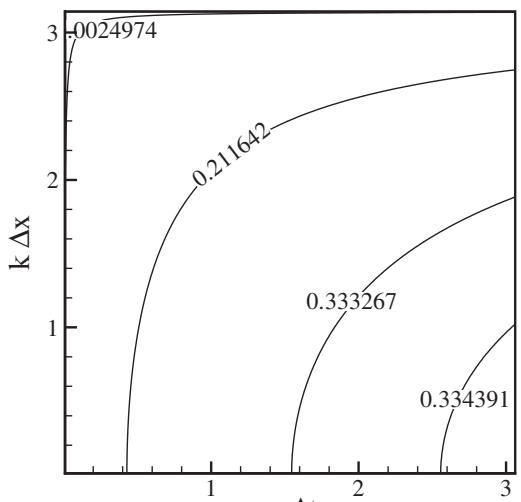

$\omega \Delta \mathrm{t}$

(b)

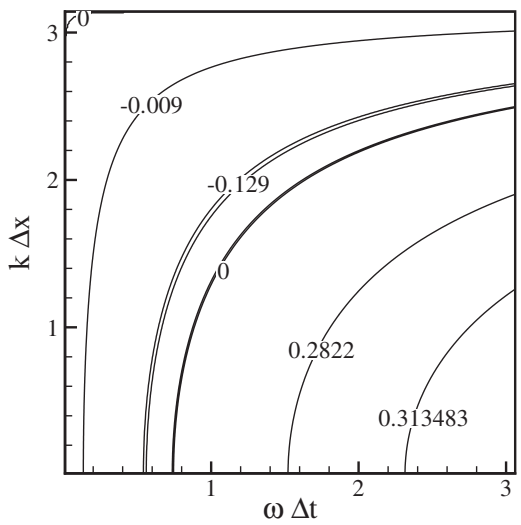

(d)

Fig. 4. The amplification factor for solving Eq. (4) using Adams-Bashforth time integration scheme and $C D_{2}$ as spatial discretization scheme. (a) The time dependent function $F_{2}$ (Eq. 14(a)) depicting the physical mode; (b) the time dependent function $\mathrm{H}_{2}$ (Eq. 14(b)) depicting the computational mode; (c) the spectral weight $M_{2}$ (Eq. 16(a)) of the physical mode; and (d) the spectral weight $N_{2}$ (Eq. 16(b)) of the computational mode.

If one replaces the second order central difference by fourth order central difference scheme, then the amplification factors $G_{4}^{\mathrm{AB}}$ can be obtained as the roots of the following quadratic

$$
G-1+i \frac{N_{c}}{2}\left(3-\frac{1}{G}\right) L=0
$$


where $L=\frac{1}{3}[4-\cos (k \Delta x)]$. The roots of the equation are

$$
\begin{aligned}
& \lambda_{1}=F_{4} e^{i \eta_{4}} \\
& \lambda_{2}=H_{4} e^{i \Gamma_{4}}
\end{aligned}
$$

where,

$$
\begin{aligned}
F_{4}(k) & =\left[C_{4}^{2}+D_{4}^{2}+2 C_{4} D_{4} \cos \left(\frac{\xi_{4}}{2}-\frac{\bar{\beta}_{4}}{2}\right)\right]^{\frac{1}{2}} \\
H_{4}(k) & =\left[C_{4}^{2}+D_{4}^{2}-2 C_{4} D_{4} \cos \left(\frac{\xi_{4}}{2}-\frac{\bar{\beta}_{4}}{2}\right)\right]^{\frac{1}{2}} \\
C_{4} & =\frac{1}{2}\left[1+\frac{9}{4}\left(N_{c} L\right)^{2}\right]^{\frac{1}{2}} \\
D_{4}= & \frac{1}{2}\left[1+\frac{81}{16}\left(N_{c} L\right)^{4}-\frac{7}{2}\left(N_{c} L\right)^{2}\right]^{\frac{1}{4}} \\
\tan \left(\bar{\beta}_{4}\right)= & -\frac{N_{c} L}{1-\frac{9}{4}\left(N_{c} L\right)^{2}} \\
\tan \left(\frac{\xi_{4}}{2}\right)= & -\frac{3}{2} N_{c} L \\
\tan \left(\eta_{4}\right)= & \frac{C_{4} \sin \left(\frac{\xi_{4}}{2}\right)+D_{4} \sin \left(\frac{\bar{\beta}_{4}}{2}\right)}{C_{4} \cos \left(\frac{\xi_{4}}{2}\right)+D_{4} \cos \left(\frac{\bar{\beta}_{4}}{2}\right)} \\
\tan \left(\Gamma_{4}\right)= & \frac{C_{4} \sin \left(\frac{\xi_{4}}{2}\right)-D_{4} \sin \left(\frac{\bar{\beta}_{4}}{2}\right)}{C_{4} \cos \left(\frac{\xi_{4}}{2}\right)-D_{4} \cos \left(\frac{\bar{\beta}_{4}}{2}\right)}
\end{aligned}
$$

And the general solution at any arbitrary time is given by

$$
U_{m}^{n}=\int M_{4}(k)\left(F_{4}\right)^{n} e^{i\left(k x_{m}-n \eta_{4}\right)} d k+\int N_{4}(k)\left(H_{4}\right)^{n} e^{i\left(k x_{m}-n \Gamma_{4}\right)} d k
$$

The time-invariant coefficients are similarly found from $U_{m}^{0}$ and $U_{m}^{1}$, as

$$
\begin{aligned}
& M_{4}(k)=A_{0} \frac{1-i N_{c} L-H_{4} e^{i \Gamma_{4}}}{F_{4} e^{i \eta_{4}}-H_{4} e^{i \Gamma_{4}}} \\
& N_{4}(k)=A_{0} \frac{-1+i N_{c} L+F_{4} e^{i \eta_{4}}}{F_{4} e^{i \eta_{4}}-H_{4} e^{i \Gamma_{4}}}
\end{aligned}
$$


In Figs. 5(a) and 5(b) the time dependent parts of the physical $\left(F_{4}\right)$ and computational mode $\left(H_{4}\right)$ contours are plotted in the $(k \Delta x-\omega \Delta t)$-plane. Similarly, in Figs. 5(c) and 5(d) the corresponding spectral weights of the initial condition, $M_{4}$ and $N_{4}$, contours are displayed. These results are qualitatively same as that for the $C D_{2}$-scheme.

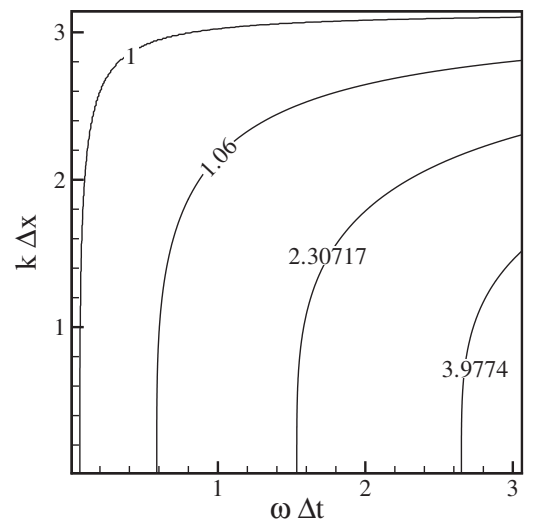

(a)

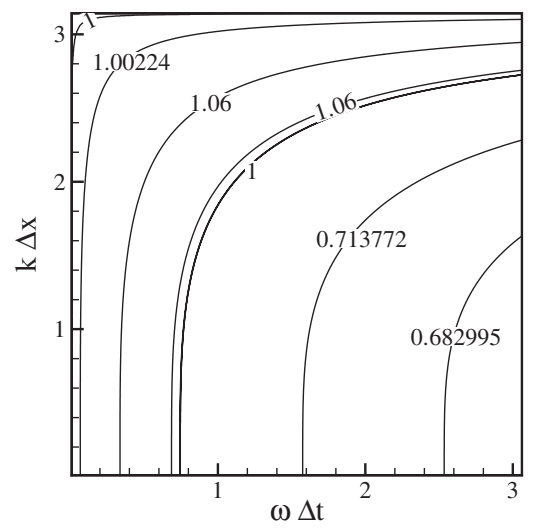

(c)

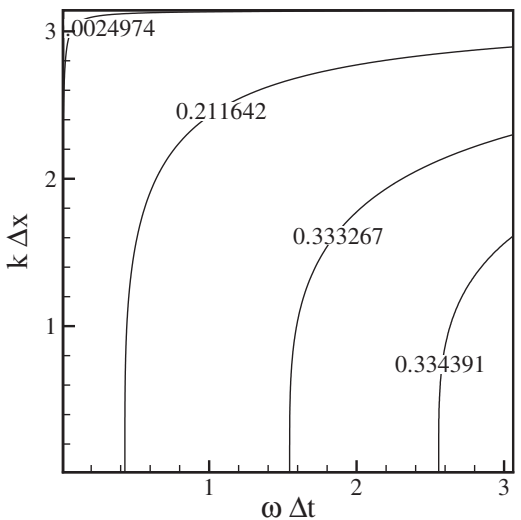

(b)

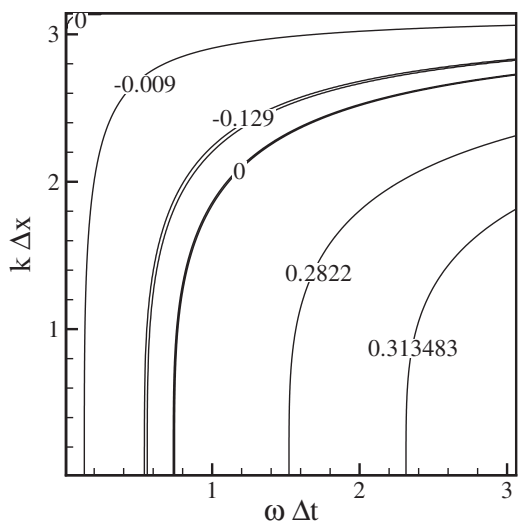

(d)

Fig. 5. The amplification factor for solving Eq. (4) using Adams-Bashforth time integration scheme and $\mathrm{CD}_{4}$ as spatial discretization scheme. (a) The time dependent function $F_{4}$ (Eq. 19(a)) depicting the physical mode; (b) the time dependent function $H_{4}$ (Eq. 19(b)) depicting the computational mode; (c) the spectral weight $M_{4}$ (Eq. 21(a)) of the physical mode; and (d) the spectral weight $N_{4}$ (Eq. 21(b)) of the computational mode. 
When the third order upwind scheme is used for $\frac{\partial u}{\partial x}$ (using Eq. (9)) in Eq. (4) along with Adams-Bashforth time integration scheme, the amplification factors are found as the roots of the following quadratic equation,

$$
G-1+i \frac{N_{c}}{2}\left(3-\frac{1}{G}\left(L_{2}+i L_{1}\right)\right)=0
$$

The roots of this equation can be written as

$$
\begin{aligned}
& \lambda_{1}=F_{3} e^{i \eta_{3}} \\
& \lambda_{2}=H_{3} e^{i \Gamma_{3}}
\end{aligned}
$$

where,

$$
\begin{aligned}
L_{1}= & -24 \sin ^{2}\left(\frac{k \Delta x}{2}\right) \\
L_{2}= & (4-\cos (k \Delta x)) \sin (k \Delta x) \\
F_{3}(k)= & {\left[C_{3}^{2}+D_{3}^{2}+2 C_{3} D_{3} \cos \left(\frac{\xi_{3}}{2}-\frac{\bar{\beta}_{3}}{2}\right)\right]^{\frac{1}{2}} } \\
H_{3}(k)= & {\left[C_{3}^{2}+D_{3}^{2}-2 C_{3} D_{3} \cos \left(\frac{\xi_{3}}{2}-\frac{\bar{\beta}_{3}}{2}\right)\right]^{\frac{1}{2}} } \\
C_{3}= & \frac{1}{2}\left[1+\frac{1}{16} N_{c}^{2}\left(L_{1}^{2}+L_{2}^{2}\right)+\frac{N_{c} L_{1}}{2}\right]^{\frac{1}{2}} \\
D_{3}= & \frac{1}{2}\left[1+\frac{N_{c} L_{1}}{3}+\frac{1}{72} N_{c}^{2}\left(11 L_{1}^{2}-7 L_{2}^{2}\right)+\frac{1}{48} N_{c}^{3} L_{1}\left(L_{1}^{2}+L_{2}^{2}\right)\right. \\
& \left.+\frac{1}{64} N_{c}^{4}\left(L_{1}^{2}+L_{2}^{2}\right)^{4}\right]^{\frac{1}{4}} \\
\tan \left(\bar{\beta}_{3}\right)= & -\frac{N_{c} L_{2}\left(\frac{1}{3}+\frac{N_{c} L_{1}}{4}\right)}{2+\frac{1}{8} N_{c}^{2}\left(L_{1}^{2}-L_{2}^{2}\right)+\frac{N_{c}^{4}}{3}} \\
\tan \left(\Gamma_{3}\right)= & \frac{C_{3} \sin \left(\frac{\xi_{3}}{2}\right)-D_{3} \sin \left(\frac{\bar{\beta}_{3}}{2}\right)}{C_{3} \cos \left(\frac{\xi_{3}}{2}\right)-D_{3} \cos \left(\frac{\bar{\beta}_{3}}{2}\right)} \\
\tan )= & -\frac{N_{c} L_{2}}{4+N_{c} L_{1}} \\
\tan \left(\eta_{3}\right)= & \frac{C_{3} \sin \left(\frac{\xi_{3}}{2}\right)+D_{3} \sin \left(\frac{\bar{\beta}_{3}}{2}\right)}{C_{3} \cos \left(\frac{\xi_{3}}{2}\right)+D_{3} \cos \left(\frac{\bar{\beta}_{3}}{2}\right)} \\
\tan & \\
\tan &
\end{aligned}
$$


The general solution in this case is given by,

$$
U_{m}^{n}=\int M_{3}(k)\left(F_{3}\right)^{n} e^{i\left(k x_{m}-n \eta_{3}\right)} d k+\int N_{3}(k)\left(H_{3}\right)^{n} e^{i\left(k x_{m}-n \Gamma_{3}\right)} d k
$$

where $M_{3}$ and $N_{3}$ are obtained as

$$
\begin{aligned}
& M_{3}(k)=A_{0} \frac{\left(1+\frac{N_{c} L_{1}}{6}\right)-H_{3} e^{i \Gamma_{3}}-\frac{N_{c} L_{2}}{6}}{F_{3} e^{i \eta_{3}}-H_{3} e^{i \Gamma_{3}}} \\
& N_{3}(k)=A_{0} \frac{-\left(1+\frac{N_{c} L_{1}}{6}\right)+F_{3} e^{i \eta_{3}}+\frac{N_{c} L_{2}}{6}}{F_{3} e^{i \eta_{3}}-H_{3} e^{i \Gamma_{3}}}
\end{aligned}
$$

In Figs. 6(a) and 6(b) the time dependent parts of the physical $\left(F_{3}\right)$ and the computational mode $\left(H_{3}\right)$-contours are plotted in $(k \Delta x-\omega \Delta t)$-plane respectively. Unlike the central schemes, here the computational mode is not negligible for any combination of $k \Delta x$ and $\omega \Delta t$. More over, there are large ranges of $k \Delta x$ and $\omega \Delta t$ for which the computational mode and hence the whole scheme is unstable. For physical mode there are only limited ranges of $k \Delta x$ and $\omega \Delta t$ over which this mode will produce acceptable near-neutral behavior. The physical mode shows instability for practically the whole range of $\omega \Delta t$ when $k \Delta x$ approaches zero. This feature of the third order upwind scheme, produces unstable results as grid is refineda feature known to users of this scheme (as in [1-3]). The computational mode in Fig. 6(b) shows that this component is unstable for large ranges of $k \Delta x$ and $\omega \Delta t$. The spectral weights of initial condition, $M_{3}$ and $N_{3}$, contours are plotted in Figs. 6(c) and 6(d) respectively. One notices that the computational mode significantly contributes to the solution. There is also a region of $k \Delta x$ and $\omega \Delta t$ over which the computational mode has negative sign.

In Figs. 7(a) and 7(c) the normalized numerical phase speed contours are shown for the Adams-Bashforth time integration scheme with $\frac{c_{N}}{c}$ lying between 0.95 and 1.05 , for $C D_{2}$ and $C D_{4}$ scheme respectively. It is seen that the time integration scheme is acceptable only within the shaded region. The computational mode contours are indicated in Figs. 7(b) and 7(d). It is seen that this mode travels at very high speed for small $\omega \Delta t$ values for all wavelengths. Thus integrating informations from Figs. 6 and 7 , one can see that the computational mode will have non-negligible spurious contributions at all length scales. 


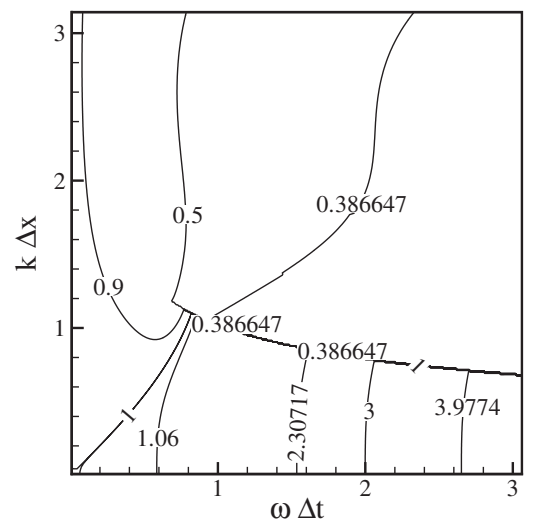

(a)

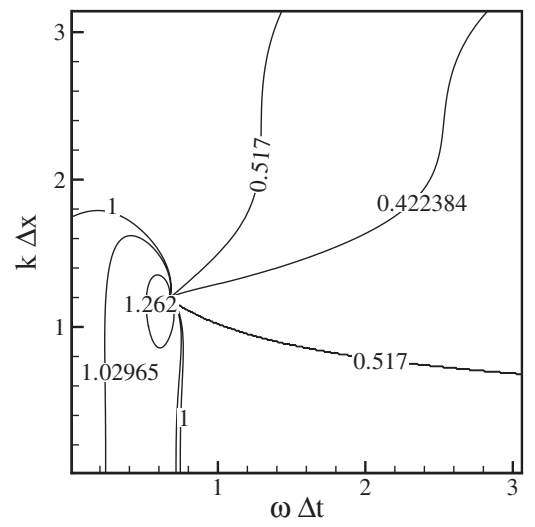

(c)

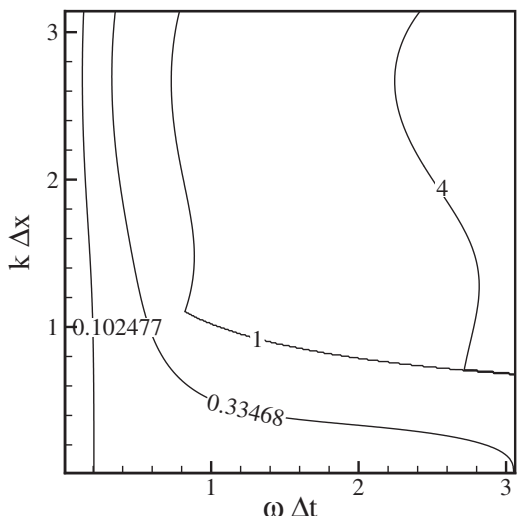

(b)

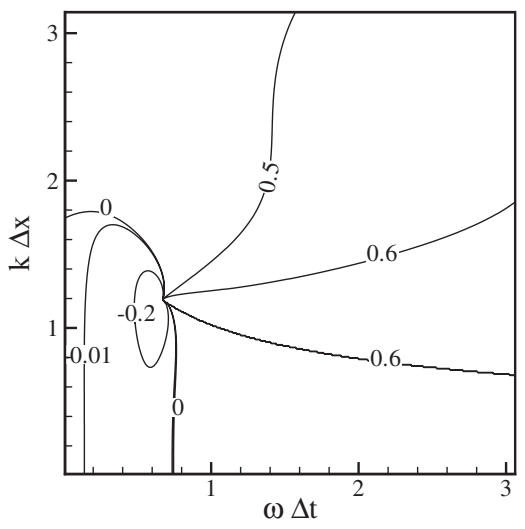

(d)

Fig. 6. The amplification factor for solving Eq. (4) using Adams-Bashforth time integration scheme and $U D_{3}$ as spatial discretization scheme. (a) The time dependent function $F_{3}$ (Eq. 24(c)) depicting the physical mode; (b) the time dependent function $\mathrm{H}_{3}$ (Eq. 24(d)) depicting the computational mode; (c) the spectral weight $M_{3}$ (Eq. 26(a)) of the physical mode; and (d) the spectral weight $N_{3}$ (Eq. 26(b)) of the computational mode.

In Figs. 8(a) and 8(b) the $\frac{c_{N}}{c}$ contours are shown for the physical and the computational modes respectively for the third order spatial upwinding scheme. The physical mode is acceptable only in the region where the contours are plotted, with the understanding that $5 \%$ tolerance is acceptable. As the constant CFL number lines pass through the origin, the patch near the origin is acceptable and the corresponding CFL numbers have to be very small. Furthermore, across the line AB in Fig. 8(b), the phase speed of the computational mode has discontinuous jump. 


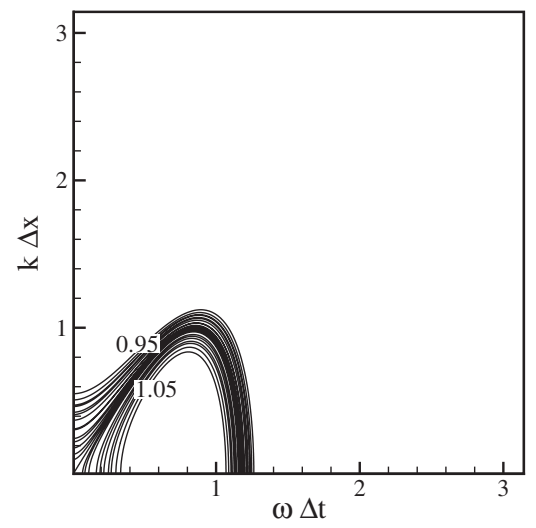

(a)



(c)

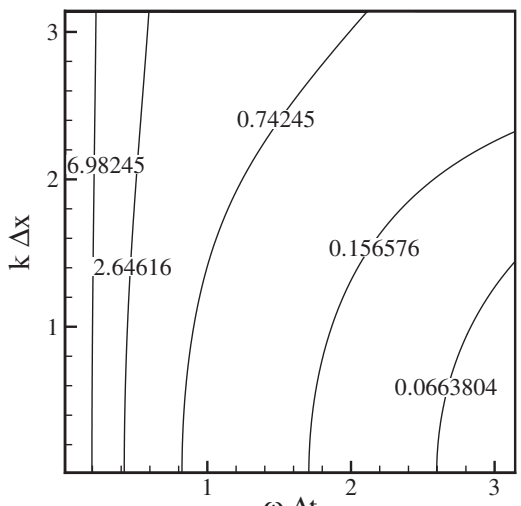

$\omega \Delta \mathrm{t}$

(b)

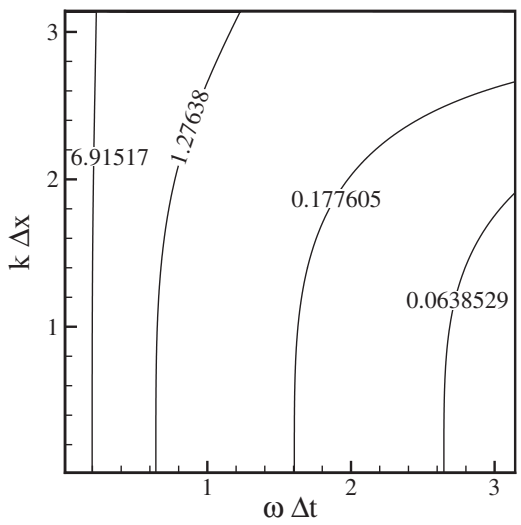

(d)

Fig. 7. The normalized numerical phase speed $c_{N} / c$ for solving Eq. (4) using AdamsBashforth time integration scheme; (a) the shaded region where $\left|\frac{c_{N}}{c}-1\right|<0.05$ for the physical mode using $C D_{2}$ scheme; (b) the contour lines $c_{N} / c$ of the computational mode using $C D_{2}$ scheme; (c) the shaded region where $\left|\frac{c_{N}}{c}-1\right|<0.05$ for the physical mode using $C D_{4}$ scheme; and (d) the contour lines $c_{N} / c$ of the computational mode using $C D_{4}$ scheme.

In Figs. 9(a) and 9(c) the scaled group velocity for the physical mode are shown for the central spatial schemes used with AB-time integration scheme. Only the shaded region near origin is acceptable where dispersion relation is preserved and the energy propagates at the correct speed. The $C D_{4}$ scheme has larger $k \Delta x$ range as compared to $C D_{2}$-scheme where this is true. The computational mode contours are plotted in Figs. 9(b) and 9(d) for $C D_{2}$ and $C D_{4}$ schemes respectively. For both the schemes, for a certain value of $k \Delta x$ the scheme displays vanishing group velocity for all frequencies. 


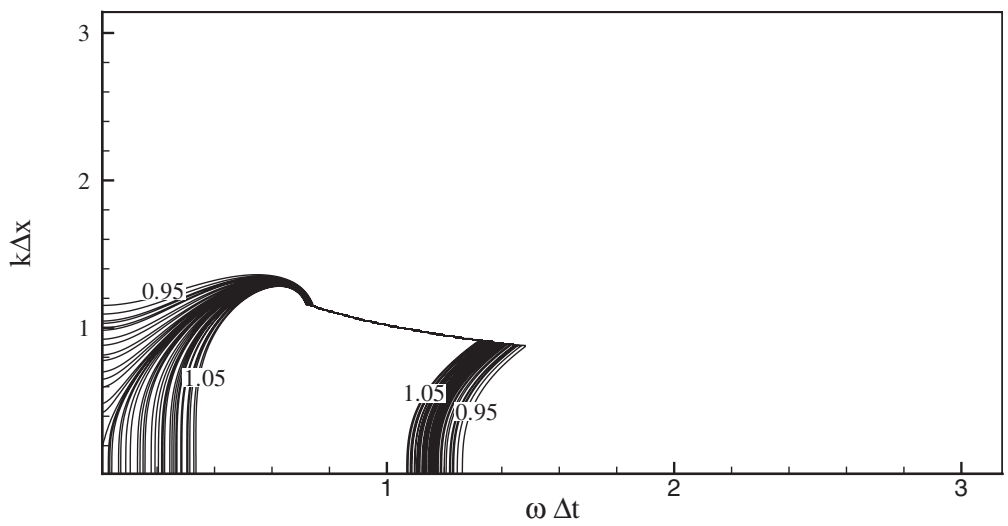

(a)

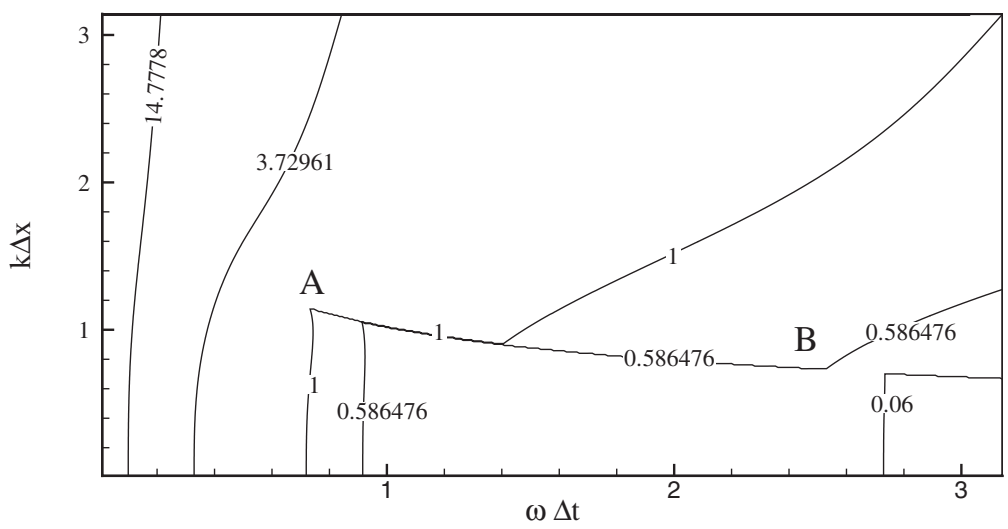

(b)

Fig. 8. The normalized numerical phase speed $c_{N} / c$ for solving Eq. (4) using AdamsBashforth time integration scheme and third order upwind spatial discretization scheme; (a) the shaded region where $\left|\frac{c_{N}}{c}-1\right|<0.05$ for the physical mode and (b) the contour lines $c_{N} / c$ of the computational mode.

Below this line, the computational mode travels upstream and hence would produce non-physical effects. Unfortunately, this is also the range where the physical mode has desirable property - as discussed above. Overall, one can see by noting Figs. 4, 5, and 9, that the choice of AB-scheme would lead to unphysical results for the central schemes.

In Fig. 10(a) the scaled group velocity of the physical mode for the third order upwind scheme is shown. This shows that larger values of $\omega \Delta t$ will produce physically relevant results, as compared to the central 


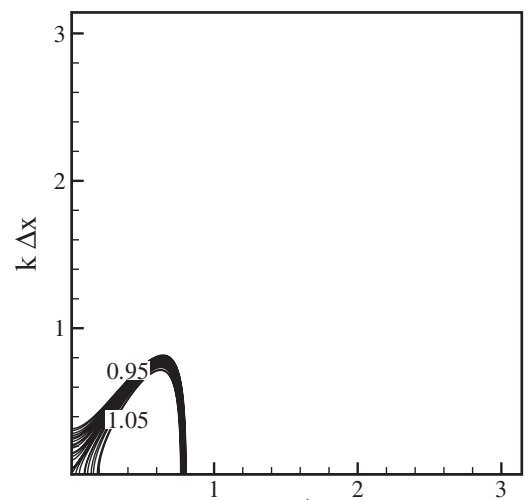

$\omega \Delta \mathrm{t}$

(a)

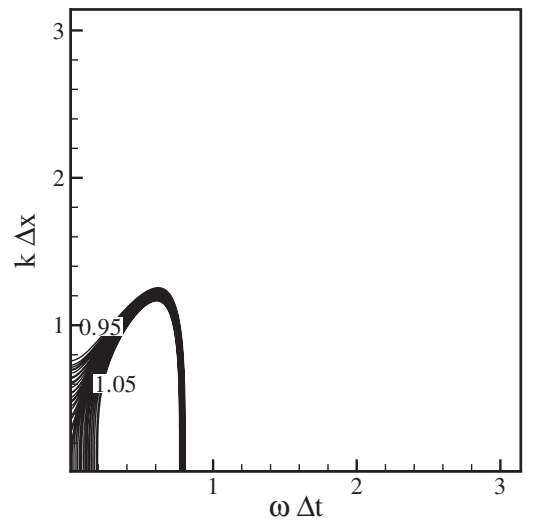

(c)

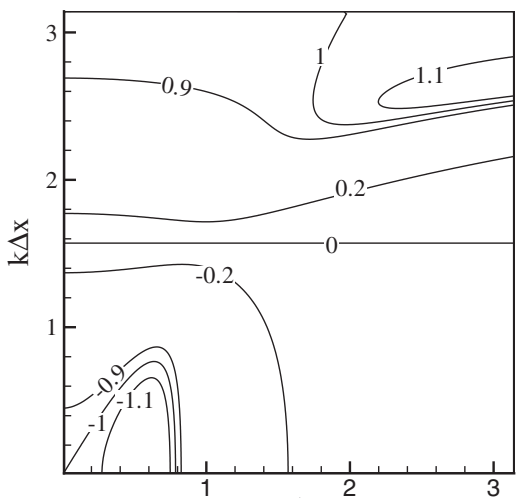

$\omega \Delta \mathrm{t}$

(b)

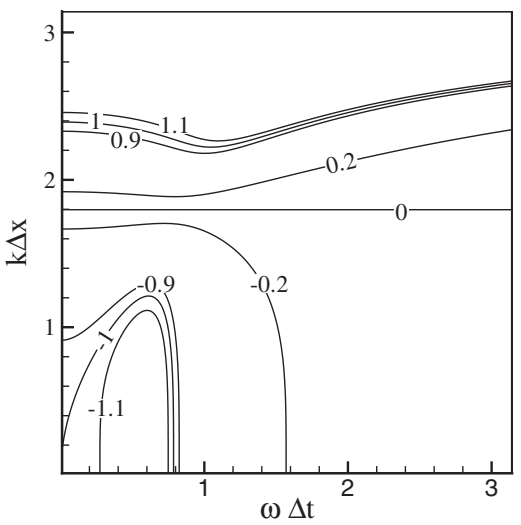

(d)

Fig. 9. The normalized numerical group velocity $V_{g N} / c$ for solving Eq. (4) using AdamsBashforth time integration scheme; (a) the shaded region where $\left|\frac{V_{g N}}{c}-1\right|<0.05$ for the physical mode using $C D_{2}$ scheme; (b) the contour lines $V_{g N} / c$ of the computational mode using $C D_{2}$ scheme; (c) the shaded region where $\left|\frac{V_{g N}}{c}-1\right|<0.05$ for the physical mode using $C D_{4}$ scheme; and (d) the contour lines $V_{g N} / c$ of the computational mode using $C D_{4}$ scheme.

schemes. This favors the usage of third order upwind scheme as compared to the equivalent higher order central schemes. Corresponding computational mode group velocity is shown in Fig. 10(b). For small $k \Delta x$ and $\omega \Delta t$, this mode travels upstream with high speed for energy propagation. Also like the central schemes this undesirable feature is for a combination of small wave numbers and frequencies where the physical mode performs well. Thus, one can see that the AB-scheme when used with the third order upwind scheme will also produce erroneous results. 


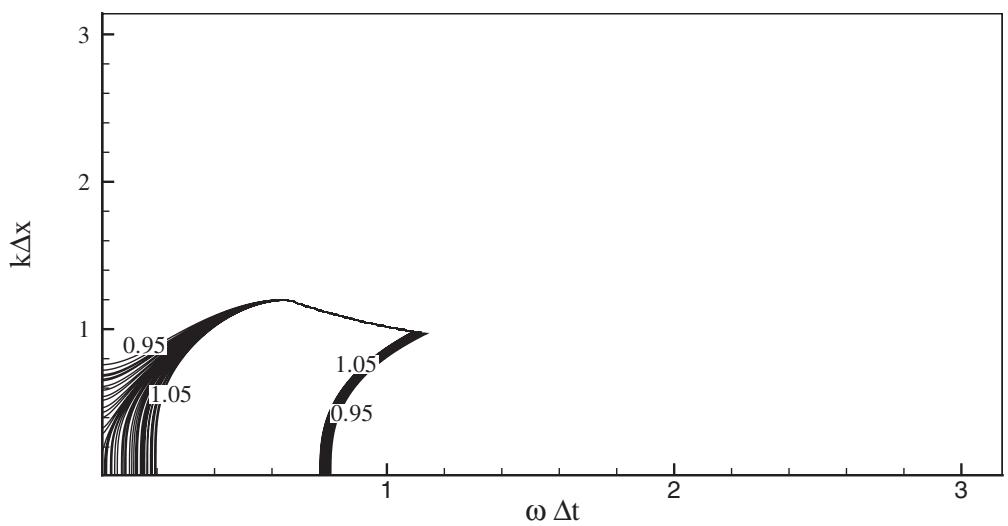

(a)

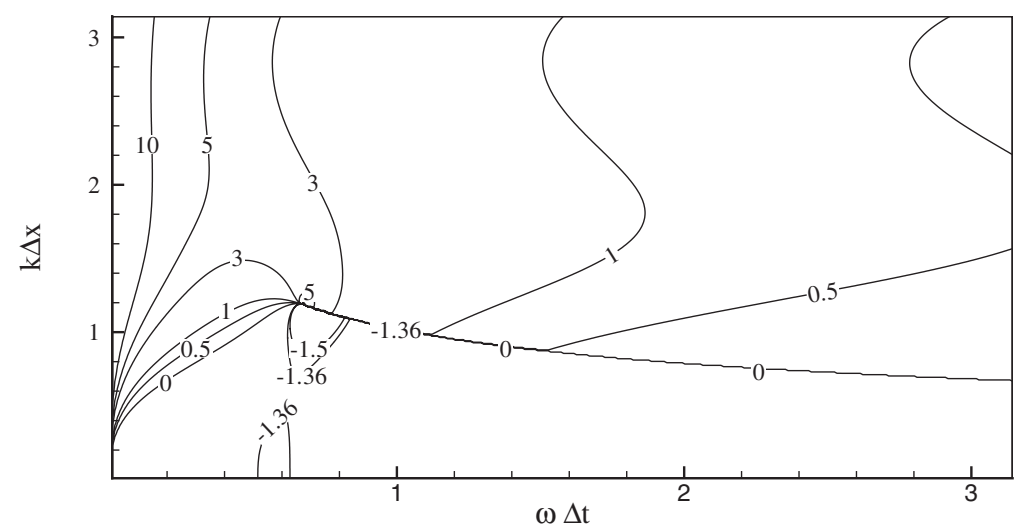

(b)

Fig. 10. The normalized numerical group velocity $V_{g N} / c$ for solving Eq. (4) using AdamsBashforth time integration scheme and third order upwind spatial discretization scheme; (a) the shaded region where $\left|\frac{V_{g N}}{c}-1\right|<0.05$ for the physical mode and (b) the contour lines $V_{g N} / c$ of the computational mode.

\section{SOLVING NAVIER-STOKES EQUATION USING THIRD ORDER UPWIND SCHEME}

The discussion in previous section reveals that the central schemes cannot be used for Euler time integration scheme due to numerical instability. This was avoided in [14] by switching over to second order $\mathrm{AB}$-scheme for time integration. However, the presence of the computational mode gives rise to spurious contributions for low wave numbers across 
all frequencies. This includes the unphysical upstream propagation of many low to mid-wave number components - as seen from Fig. 9.

As compared to central schemes third order upwind scheme has appreciable range of $k \Delta x$ and $\omega \Delta t$ for which the scheme has better dispersion relation preservation property for Euler time integration scheme. This is clearly seen by comparing Fig. 3(c) with Figs. 3(a) and 3(b). However, one is restricted to very small time steps due to numerical stability consideration - as can be seen from Fig. 1(c). In contrast, if $\mathrm{AB}$-scheme is used for time integration, the physical mode allows taking comparatively larger time steps - as evident in Fig. 6(a) in comparison to Fig. 1(c). However, for problems where a range of frequencies are excited - as in flow problems involving instabilities and turbulence - this method will still require taking very small time steps due to numerical instability consideration. Additionally, in this case the computational mode is not negligible as compared to central schemes. This is readily apparent by comparing Figs. 6(b) and 6(d) with Figs. 4(b), 4(d), 5(b), and 5(d). While the computational mode is always stable for the central schemes (as seen in Figs. 4(b) and 5(b)), for the upwind scheme the computational mode is unstable for high frequencies and high wave numbers, as seen in the right hand corner region in Fig. 6(b). Furthermore, a comparison between Figs. 9 and 10 reveal that the computational mode travel upstream across a large frequency band for physically relevant small wave numbers. This nonphysical upstream propagation of energy will make the solution incorrect.

These observations are demonstrated here by solving Navier-Stokes equation for flow past a rotating circular cylinder using the third order upwind scheme for spatial discretization and Euler and AB-schemes for temporal discretization. The physical problem is chosen for flow at a Reynolds number of $\mathrm{Re}=3800$ (based on diameter and free-stream speed as length and velocity scales) and a non-dimensional rotation rate of $\Omega=10$, i.e., when the peripheral speed of the cylinder is ten times the freestream speed. Flow past rotating cylinder for this type of flow-parameters display temporal physical instabilities - as reported in the experiments of [27]. This has also been observed computationally by high accuracy schemes for Navier-Stokes equation and theoretical explanation provided for the observed temporal instabilities in [28] by using third order upwind scheme for spatial discretization and Euler time marching scheme, using stream function-vorticity formulation for $\mathrm{Re}=3800$ and $\Omega=5$. Other references are cited in [28] where results for other Reynolds numbers and rotation rates were obtained and reported earlier. In these calculations it was noted that the flow suffered temporal instabilities at discrete times after impulsive and non-impulsive start-ups. During these instabilities loads and moment change abruptly. For the present case of larger rotation rate 
$(\Omega=10)$, the lift and drag coefficients variation with time are shown in Fig. 11, where Euler and AB-schemes have been used for time-marching the vorticity transport equation. For the details of numerical methods the reader is referred to [28]. For the present very high rotation rate case, Euler time integration once again displays temporal instabilities at discrete times, as was shown in [28] for lower rotation rates and lower Reynolds numbers.

This instability was shown in [28] to arise from a receptivity mechanism where a given equilibrium flow (not necessarily a time-independent

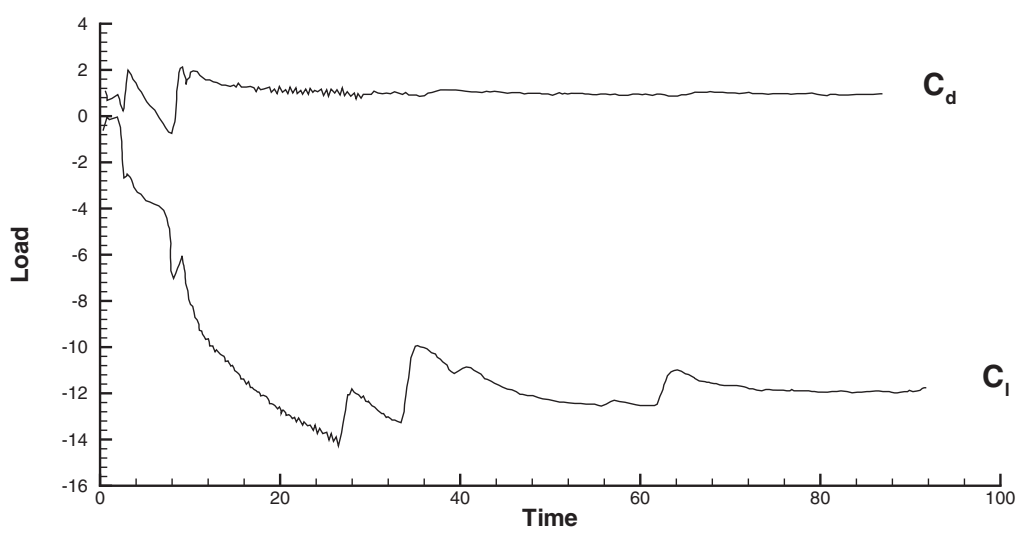

(a)

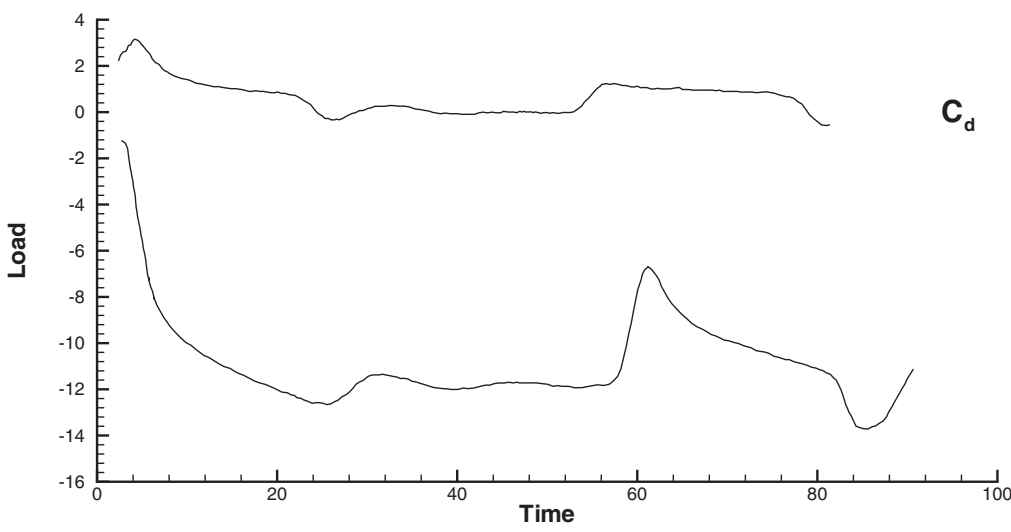

$\mathbf{C}_{1}$

(b)

Fig. 11. The calculated lift and drag coefficients for $\operatorname{Re}=3800$ and $\Omega=10$ as a function of time for impulsive start case using (a) Euler time integration scheme and (b) AdamsBashforth time integration scheme. 
flow) is destabilized by infinitesimal far-field disturbances and as a consequence a lump of vorticity that is confined within the recirculating fluid around the cylinder is released in the wake of the cylinder. For high rotation rate cases, there is a layer of fluid fluid close to the cylinder surface rotates along with the cylinder. This is distinguished from the surrounding flow by an interior layer of fluid that encompasses the full saddle point. With time, circulation of flow inside the co-rotating region near the surface of the cylinder keeps increasing. This is the reason for the increase in lift seen in Fig. 11. This unsteady growth is punctuated by the instability of the flow in the interior layer and as a consequence a large amount of vorticity is released from the co-rotating layer to the wake of the cylinder. This happens almost instantaneously. To capture such instability, it is natural that one should take very small time steps. This requirement matches perfectly well with the numerical stability requirement of the third order scheme discussed above for the Euler time-marching strategy. However, when the $\mathrm{AB}$-scheme is used for time-marching, the present computational mode with negative group velocity for combinations of small values of $k \Delta x$ and $\omega \Delta t$, will slow down or even prevent vorticity to be released in the wake. Additionally, the AB-scheme itself produces numerical dissipation via the channelizing the initial condition to the computational mode that is subsequently damped. As a consequence the instabilities are weakened and in the $C_{l}$ and $C_{d}$ vs time plots the discrete jumps in the value are smoothed out. It is to be realized that the observed instabilities occur over a time scale that is of the order of micro seconds and in non-dimensional units it is of the order of $10^{-5}$ - the time step taken for Euler time integration scheme.

This is a demonstration of the superiority of Euler time integration scheme over Adams-Bashforth time integration scheme for capturing flow instability. For DNS the excited length and time scales are even wider and the noted feature of the time integration scheme will be felt even more acutely. The present study shows the spurious behavior of AB-scheme in solving time dependent problems where large ranges of length and time scales are excited. But the seemingly stabilizing effects of $\mathrm{AB}$-scheme has lulled many CFD practitioners in adopting this time integration scheme and the aim of the present study is to discourage such practices.

\section{CONCLUSION}

In this work we have compared first the performances of Euler and Adams-Bashforth time integration schemes by looking at the analytical solution of one-dimensional wave equation. Subsequently, we have solved two-dimensional Navier-Stokes equations for flow past a very rapidly 
rotating cylinder that displays physical temporal instabilities [27, 28]. It is shown that the Euler time integration scheme captures these instabilities quite adequately when very small time steps are chosen, but the AB-scheme either smooths them or mis-predict the instabilities. Hence AB-scheme is not suitable for problems that display excitation of large bands of length and time scales, as in DNS. Instead it is preferable to use explicit Euler time integration scheme with very small time steps.

\section{REFERENCES}

1. Kawamura, T., Takami, H., and Kuwahara, K. (1985). A new higher order upwind scheme for incompressible Navier-Stokes equation. Fluid Dynam. Res. 1, 145.

2. Sengupta, T. K., and Sengupta, R. (1994). Flow past an impulsively started circular cylinder at high Reynolds number. Comput. Mech. 14(4), 298.

3. Nair, M. T., and Sengupta, T. K. (1997). Unsteady flow past elliptic cylinders. J. Fluids Struct. 11, 555.

4. Harlow, H. H., and Welch, J. E. (1965). Numerical calculation of time-dependent viscous incompressible flow of fluid with free surface. Phys. Fluids 8, 2182.

5. Lele, S. K. (1992). Compact finite difference schemes with spectral-like resolution. J. Comput. Phys. 103, 16.

6. Adams, N. A., and Shariff, K. (1996). A high resolution hybrid compact-ENO scheme for shock turbulence interaction problems. J. Comput. Phys. 127(1), 27.

7. Sengupta, T. K., Anuradha, G., and De, S. (2003). Navier-Stokes solution by new compact schemes for incompressible flows. Paper to be presented at second MIT conference on Computational Fluid and Solid Mechanics, Bathe, K. J. (ed.).

8. Sengupta, T. K., and Nair, M. T. (1999). Upwind schemes and large eddy simulation. Internat. J. Numer. Methods Fluids 31, 879.

9. Ames, W. F. (1977). Numerical Methods for Partial Differential Equations, 2nd ed., Academic Press, New York.

10. Haltiner, G. J., and Williams, R. T. (1980). Numerical Prediction and Dynamic Meteorology, Wiley, New York.

11. Lilly, D. K. (1965). On the computational stability of numerical solutions of time-dependent non-linear Geophysical fluid dynamics problems. Mon. Weather Rev. 138, 11.

12. Oran, E. S., and Boris, J. P. (2001). Numerical Simulation of Reactive Flows, 2nd ed., Cambridge University Press.

13. Durran, D. R. (1999). Numerical Methods for Wave Equation in Geophysical Fluid Dynamics, Springer-Verlag, New York.

14. Kim, J., and Moin, P. (1985). Application of fractional step method to incompressible Navier-Stokes equation. J. Comput. Phys. 58, 308.

15. Kawamura, H., Ohsaka, K., Abe, H., and Yamamoto, K. (1998). DNS of turbulent heat transfer in channel flow with low to medium-high Prandtl number. Internat. J. Heat Fluid Flow 19, 482.

16. Kawamura, H., Abe, H., and Matsuo, Y. (1999). DNS of turbulent heat transfer in channel flow with respect to Reynolds and Prandtl number effect. Internat. J. Heat Fluid Flow 20, 196.

17. Maass, C., and Schumann, U. (1994). Numerical simulation of turbulent flow over a wavy boundary. In Voke, P. R., Kleiser, L., and Chollet, J.-P. (eds.), Direct and Large Eddy Simulation, p. 287. 
18. Clerex, H. J. H. (1997). A spectral solver for the Navier-Stokes equation in the velocityvorticity formulation for flows with two non-periodic direction. J. Comput. Phys. 137, 186.

19. Boersma, B. J., Brethonwer, G., and Nieuwstadt, F. T. M. (1998). A numerical investigation on the effect of the inflow conditions on the self-similar region of a round jet. Phys. Fluids 10, 899.

20. Serre, E., Bontonx, P., and Kotorba, R. (2001). Numerical simulation of transition in three-dimensional rotating flows with walls: Boundary layer instability. Int. J. Fluid Dynam. 5(2), 17.

21. Karamanos, G. S., and Karniadakis, G. E. (2000). A spectral vanishing viscosity method for large eddy simulations. J. Comput. Phys. 163, 22.

22. Sipp, D., Jacquin, L., and Cosssu, C. (2000). Self-adaption and viscous and selection in concentrated two-dimensional vortex dipoles. Phys. Fluids 12(2), 245.

23. Perot, J. B. (1992). DNS of turbulence in connection machine. In Pelz, R., Ecer, A., and Hauser, J. (eds.), Proceedings of Parallel CFD-1992, Elsevier, The Netherlands.

24. Lam, K., and Banerjee, S. (1992). On the condition of streak formulation in bounded flow. Phys. Fluids 4, 306.

25. Shi, J., Thomas, T. G., and Williams, J. J. R. (2000). Free surface effects in open channel flow at moderate Froude and Reynolds number. J. Hydraul. Res. 38(6), 465.

26. Sengupta, T. K., Ganerwal, G., and De, S. (2003). Analysis of central and upwind compact schemes. J. Comput. Phys., accepted for publication.

27. Werle, H. (1984). Hydrodynamic visualization of the flow around a streamlined cylinder with suction. Cousteau-Malavard sail model, Le Rechereche Aerospatiale 4, 29.

28. Sengupta, T. K., Kasliwal, A., De, S., and Nair, M. T. (2003). Temporal flow instability for Magnus-Robins effect at high rotation rates. J. Fluids Struct. 17(7), 941. 